\title{
How number is associated with space? The role of working memory
}

Wim Fias $^{1}$

Jean-Philippe van Dijck ${ }^{1}$

Wim Gevers ${ }^{2}$

${ }^{1}$ Department of Experimental Psychology, Ghent University, Belgium

${ }^{2}$ Unité de Recherches en Neurosciences Cognitives, Université Libre de Bruxelles, Belgium

Corresponding author:

Wim Fias

H. Dunantlaan 2

B-9000 Gent

Belgium

wim.fias@ugent.be 


\begin{abstract}
A large body of evidence demonstrates that the processing of numbers and space are tightly related. Today, the dominant explanation for this interaction is the number line hypothesis. This hypothesis claimis that the mental representation of numbers takes the form of a horizontally oriented line which is functionally homeomorphic to the way physical lines are represented. The aim of the present review is twofold. In a first step we review recent evidence on number bisection bias in neglect and on the SNARC effect challenging the homeomorphic relationship between numerical and spatial processing. For the bisection bias we present data that show a clear dissociation between number bisection and physical line bisection. Additionally, we present data on the SNARC effect showing that its origin is of a conceptual rather than visuospatial nature. In a second step, we present data that provide pointers towards a new theoretical framework which proposes that serial position in working memory may be an important determinant of the interactions between number and space.
\end{abstract}




\section{How number is associated with space? The role of working memory}

It is generally accepted that the cognitive representation and processing of number and space are tightly linked. This is evident from introspective reports, for instance by mathematicians who describe their mathematical thinking as hinging on visual imagery. Also, math instruction and education rely strongly on visuospatial tools and strategies. Remarkably, the involvement of spatial processing in mathematical cognition is not restricted to complex mathematics but even applies to the basic and elementary representation of number.

Already in the 1880's Galton [e.g. 1] described the introspective reports of people who experience vivid mental number line images. Later, Seron et al. [2] showed that these were more than incidental observations and confirmed that about $14 \%$ of typical student population report mental number lines. Experiences of spatially defined mental number lines can be particularly strong and elaborated in synesthesia [3,4], a condition in which certain types of stimuli give rise to experiences in modalities that are normally not associated with such stimuli, for instance color sensations to number stimuli. Importantly, however, number-space interactions are not restricted to those who have the conscious experience of mental number lines. A number of empirical phenomena demonstrate that the involvement of space is a basic and essential aspect of number representation also in people who do not report having conscious experience of visuospatial number lines when they think about numbers.

In a seminal paper, Dehaene et al. [5] showed that, when subjects respond to numbers by pressing a left or a right response key, for instance in a parity judgment task, small numbers are faster responded to with the left hand than with the right hand and that large numbers receive faster right hand than left hand responses. This effect (see Figure 1) indicating a Spatial Numerical Association of Response Codes (SNARC effect) has since then been replicated numerous times with paradigms using different stimulus and task configurations [for a review see 6].

Another phenomenon, for the first time reported by Zorzi et al. [7] is observed in many brain damaged patients suffering from spatial hemineglect, most frequently after right hemisphere 
lesion. These patients fail to report, orient to, or verbally describe stimuli in the contralesional left hemispace [for a review see 8], as is for instance evident from tests like physical line bisection where patients typically mark their perceived midpoint right from the veridical midpoint. When these patients are asked to name the number that is in the middle of a numerical interval (e.g. Which number is in the middle between 1 and 9?), they often produce a number that is larger than the true middle number, whereby the size of the bias is positively correlated to the size of the difference between the two numbers. Together with a cross-over effect (i.e. a tendency of a bias towards smaller numbers in small numerical intervals) the pattern of number bisection bias strongly resembles the bias observed when neglect patients bisect physical lines (see Figure 2).

While the idea of a mental number line was originally conceived of as a metaphor [e.g. 9], the strong similarity between number and physical line bisection has been taken as evidence in favor of a stronger version of the mental number line hypothesis that argues that the mental number line is not simply a metaphor but that the mental representation of numerical magnitude is considered to be homeomorphic to the representation of physical space [e.g. 10], with a common mechanism of spatial attention operating on both numerical and physical space [e.g. 7, 11]. The hypothesis of a homeomorphic mental number line has since become the dominant theoretical framework to account for various instances of number-space interactions [for review see for instance $12,13,14]$.

In what follows, we will review recent evidence on number bisection bias in neglect and on the SNARC effect that challenges this interpretation. In doing so, we will provide pointers towards an alternative framework that proposes serial position in working memory as an important determinant of the interactions between number and space. 


\section{Dissociating the number interval bisection bias from the line bisection bias}

The parallellism between number interval bisection and physical line bisection in neglect offers a high degree of face validity to the mental number line hypothesis. Just like patients ignore the left side of physical space and therefore produce a rightward bias in line bisection, the patients are assumed to ignore the left side of the number line which then leads to a bisection towards the right side of the number line where the large numbers are located.

However, the idea of a functional isomorphism between bisection of physical and number space has been questioned. Doricchi et al. [15, for a larger followup study see 16] observed a double dissociation between number interval bisection and physical line bisection. Those patients that established a strong bias in line bisection produced only a weak or no bias in number bisection and vice versa. This suggests that both tasks depend on at least partly dissociable mechanisms. Interestingly, Doricchi et al. observed the number interval bisection bias to occur only in those patients whose lesion comprised prefrontal cortex [15] and whose visuospatial and verbal working memory span was reduced [16]. This suggests that working memory span may play a crucial role in the number interval bisection task. However, on itself, a reduced span can explain generally inaccurate performance in the number interval bisection task, but is not sufficient to account for the systematic deviation towards the larger numbers.

Recently, van Dijck et al. [17] presented a single-case study in which a clear dissociation between number and line bisection was present within the same patient. Moreover, the performance of the patient was informative for how a working memory deficit can give rise to a systematic deviation towards larger numbers. This patient had an ischemic left hemisphere lesion mainly affecting temporo-parietal areas and extending into frontal cortex and accordingly exhibited a rightsided neglect for physical space (as tested with tasks like physical line bisection) as well as for representational space [as tested with the o'clock task, 18, and the description of a memorized picture, 19]. Surprisingly, the patient showed a number interval bisection bias towards the larger numbers, just like all other reports of number interval bisection bias after right hemisphere lesion 
and left-sided neglect. Thus, while physical line bisection was biased to the left because of her right neglect, number interval bisection biased remained directed towards the larger numbers. The possible explanation that this was due to a reversed mental number line (i.e. from right to left) was ruled out by the fact that the patient showed a normal SNARC effect, (faster left than right hand responses to small numbers and vice versa for large numbers).

Interestingly, a close examination of the patient's working memory performance revealed a pattern that can explain the bisection bias. While her visuospatial memory span fell within the normal range, her verbal memory span was reduced to only 3 items, as measured with a forward letter span task. By means of a probe recognition task that measured the capacity of verbal working memory at different positions within the serial sequence to be remembered, it was found that the patient was specifically impaired in remembering items from the beginning of the working memory sequence (running against the primacy effect typically observed in working memory). Furthermore, with the use of a position recall task (where the patient had to reproduce the element at a specific position in the sequence), the nature of the errors was investigated. It was found that errors were primarily items that were positioned further in the sequence than the requested position. Clearly this position-specific impairment in verbal working memory provides a meaningful explanation for the patient's number bisection bias. After all, when the initial numbers of the to-be bisected numerical sequences are not efficiently kept in mind and the bisection is performed on the remaining numbers, a shift towards the larger number is a logical consequence.

The involvement of verbal working memory in the bisection performance of this patient is further supported by the fact that the same directional bias that was observed in number interval bisection also occured in a letter interval bisection (e.g. Which letter is in the middle between A and E?) and in a word bisection task (e.g. Which is the middle letter of the word 'voltage'?). 
Although the experiments in this patient do not allow pinpointing the exact processes and representations involved in number interval bisection, they do allow concluding that number interval bisection bias can, despite its apparent similarity with physical physical line bisection, completely dissociate from it and even go in the opposite direction. It is true of course that generalizing from a single case study is not without problems, but the fact that position-specific working memory deficit can give rise to biased number interval bisection performance urges to seriously consider the involvement of working memory in future neuropsychological studies.

Of course, generalisation of the interpretations from a single case study is not without problems. Therefore, van Dijck et al. [20] ran a group study evaluating whether number and physical line bisection depend on a shared spatial attention mechanism by exploring the extent to which both correlate. Right-hemisphere damaged neglect patients $(n=9)$, patients with right hemisphere lesion without neglect $(n=5)$ and healthy age-matched controls $(n=12)$ were included. All subjects were submitted to both the physical physical line bisection and the number interval bisection task. In addition, the SNARC effect was measured in both a parity judgment and a number comparison task. The results showed that only the neglect group showed a bisection bias in line and number interval bisection, as expected. All groups showed a SNARC effect in the parity judgment and in the number comparison tasks. The effects (bias for bisection tasks and the SNARC slopes for the parity judgment and the comparison tasks) were subjected to a principal component analysis. From the mental number line account, it is predicted that a single factor component would be sufficient to capture the correlational structure between the space-related effects obtained in the different tasks. However, the PCA revealed that such solution is not the case. The results showed that a three-component solution provided the best fit of the data pattern. The first component was loaded by magnitude comparison and number interval bisection, the second component by parity judgment and number interval bisection and the third component only by physical line bisection.

This result clearly refutes the strong version of the mental number line hypothesis by showing that multiple factors are needed to capture the correlations between the tasks. However, 
given the fact that PCA is not well suited to determine the details of underlying mechanisms (especially when the number of subejcts is relatively limited), further conclusions are not warranted. Nevertheless, both the single case and the group study demonstrate that a critical stance is needed towards the mental number line hypothesis as the sole explanatory mechanism of how number relates to space. In the following, we will focus on the SNARC effect and ask two questions. First, can the SNARC effect be dissociated from the mental number line explanation? Second, if it can be dissociated, then the question to the origin of the SNARC effect is open. Following the suggestions just described, the involvement of working memory in the SNARC effect will be explored.

\section{Dissociating SNARC from the number line: Conceptual versus visuospatial representations}

People acquire knowledge about the world through sensory-motor experience. Hence, it is no wonder that high level cognition is grounded in sensorimotor experience [21] and that mental representations of this knowledge are at least to some extent analogous to the world they represent. Yet, it is also true that humans are a communicative species that use language to communicate thoughts and cognition with their conspecifics, the essential characteristic of language being that it summarizes information in structured and labeled categories. Hence, humans are also exposed to knowledge in a format that is abstracted away from direct physical reality. This makes it realistic to assume that information is not only represented in an analogue way but also in a format that makes abstraction of instances of sensory-motor experience. This distinction is developed in the dual coding theory of Paivio [22] who claims that information is coded in an analogue system and in a verbal-symbolic system. Along the same lines, but in the specific context of spatial representations, Kosslyn et al. [23, 24] distinguished between categorical and coordinate spatial representations. The coordinate system codes for spatial position in a quantitatively precise way and the categorical 
system defines space in more qualitative and conceptually distinct classes (for instance, left vs right, or above vs below).

The mental number line hypothesis envisages only associations with the analogue representational system: Numbers are positioned on a mental number line that is defined in the same metrics as physical space is mentally coded $[25,7,12]$. As such it can account for the number interval bisection bias in neglect and for the SNARC effect. Yet, recently it has been argued that the dimensional overlap between number and response location in the SNARC effect is not necessarily located at a visuospatial level but can also be situated at a categorical level, i.e. at a level of spatial representation that is not analogous to physical space but that is tightly linked to language. In this respect, Proctor et al. [26] developed the theoretical idea that the SNARC effect derives from a systematic association between the verbal concepts such as small and left and large and right based on the assumption that conceptual categorical dimensions (like left-right and small-large) have a specific polarity (e.g. left being negative and right positive; and similarly small being negative and large being positive). According to the polarity coding account it is the congruency between these polar codes that drives the SNARC effect. Similarly, but in a computationally explicit way, Gevers et al. [27] argued that the SNARC effect results from learned connections between a number's conceptual categorisation as small or large and the conceptual category of the response (e.g. left or right).

A first empirical indication that the SNARC effect does not imply the existence of a mental number line was observed by Santens and Gevers [28]. Here, participants performed a regular magnitude comparison task with a fixed reference (i.c. 5), but instead of left-right responses, participants had to respond to a location that was close to or far from the initial finger position. On the mental number line, the numbers 4 and 6 are close to the number 5 whereas the numbers 1 and 9 are far from this reference number. Therefore, a direct mapping between the number line and the position of the response would result in faster 'close' than 'far' responses for the numbers 4 and 6 and faster 'far' than 'close' responses and the numbers 1 and 9. This was not observed. Small 
numbers ( 1 and 4$)$ were fastest with the close response and large numbers (6 and 9) with the far response. This result is in line with a conceptual account that systematically associates small to close and large to far, following Proctor et al. [26].

Gevers et al. [29] followed up on this idea and designed an experimental paradigm that allowed to determine the categorical versus visuospatial nature of the SNARC effect. The essential characteristic of this paradigm is that response buttons are variably (from trial to trial) labeled by response defining words (e.g. the label 'left' and the label 'right'). For instance, in a parity judgment task, subjects were asked to press the button wearing the label "left" if the number was even or press the button labeled "right" if the number was odd. In doing so, Gevers et al. could track whether the SNARC effect followed the visuospatial position of the hands emitting the response or the categorical concepts expressed by the labels. Both visuospatial and categorical coding could be observed when tested separately, but when both coding systems were directly pitted against one another, a pronounced dominance of the concepts expressed by the labels was observed, both in parity judgment and in number comparison. This observation provides direct evidence that numbers can interact with spatial representations at a conceptual level and that these interactions are the dominant ones for the SNARC. These results are difficult to reconcile with the homeomorphic mental number line hypothesis, because the conceptual nature of the underlying representation radically differs from the visuospatial coordinate system that is assumed to define the mental number line. At the same time, this implies that the idea of the involvement of a spatial attention mechanism in the SNARC effect is difficult to maintain.

\section{The SNARC effect: A crucial role for Working Memory}

A number of research reports have shown that the associations between number and space are not absolute but depend on contextual aspects of the stimuli, the responses and the task. This might 
suggest that the SNARC effect does not depend so much on long term associations between numbers and space, as implied in the mental number line hypothesis, but rather on temporary representations.

First, the association between a specific number with left or right depends on the range in which that number occurs $[5,30]$. For instance, number 5 presented in the context of numbers ranging from 1 to 5 will receive faster right than left hand responses, but when that same number 5 occurs in the range 4 to 9 it will receive faster left than right hand responses. Similarly, when subjects are asked to envisage the numbers as being displayed on a clock face, the SNARC effect reverses, following the fact that now small numbers occur on the right side of the clock face and large numbers on the left side fo the clock [31].

Second, the fact that the SNARC effect depends on response characteristics is shown by the fact that the left-right SNARC effect only occurs when responses have to be discriminated along the left-right dimension, suggesting that spatial coding needs to be part of the response or task set [32]. This was convincingly demonstrated by the experiments of Notebaert et al. [33] who showed that the SNARC effect can actually be reversed by creating new short term memory associations between numbers and side of response. These associations were created by means of an inducer task where the letter $\mathrm{X}$ was presented either on the left or on the right side of fixation. Participants had to respond incompatible to the location of the letter X (e.g. press left if it appears on the right side, press right if it appears on the left side). In this way new associations are created between the position 'left' and the response 'right' and between the position 'right' and the response 'left' [see also 34]. The interest was to observe what these newly created associations would do with an intermixed SNARC task (e.g. centrally presented numbers that had to be judged on their orientation). The results showed that by creating these new short term associations, the SNARC effect reversed. The effect of these short term associations suggests an important role for working memory. 
Third and relatedly, it has been shown that Russian-Hebrew bilinguals show a normal SNARC effect when they had read a Russian text shortly before but a reversed SNARC effect when having read a Hebrew text before [35]. Similarly, Shaki and Gevers [36] showed that, in Israelian participants, the SNARC effect can reverse simply on the basis of the task instruction. Hebrew letters contain both ordinal (e.g. B comes after A in the alphabet) and magnitude meaning (e.g. the letter B is 2). When asked to judge the letters for their ordinal meaning, a SNARC effect was observed in line with their reading and writing direction (letters from the beginning of the alphabet - right response / letters far in the alphabetic sequence - left response). However, when asked to judge the letters on their magnitude meaning a left-to-right SNARC effect was observed.

Observations such as these might indicate that numbers are not intrinsically related to space but that the relation is constructed during task execution, which might suggest a crucial role for working memory. This has been tested by evaluating the effect of working memory load on the SNARC effect. Herrerra et al. [37] and van Dijck et al. [38] showed that the SNARC effect in magnitude comparison disappears when visuospatial working memory is loaded (by Corsi block configurations). Van Dijck et al. additionally showed that in parity judgement it is verbal working memory load that abolished the SNARC effect. This indicates that the SNARC effect critically depends on the availability of working memory resources. Additionaly, the results suggest that depending on the task verbal or visuospatial working memory are recruited.

Of course, just like a reduced working memory capacity is on itself not sufficient to explain the number interval bisection bias in neglect, the necessity of free working memory resources for the SNARC effect to occur does on itself say nothing about the nature of the underlying mechanism. Although such an association has never been reported, it might be plausible to assume that serial position in working memory is associated with left and right, with items in the initial 
positions being associated with left and items towards the end of the working memory sequence being associated with right.

To test this hypothesis, van Dijck and Fias [39] designed an experimental procedure that allowed to dissociate the effects of position in a working memory sequence from position on a mental number line as a function of number magnitude. Subjects were presented with a randomly ordered series of numbers and were asked to remember these numbers to reproduce them later in correct order. During the retention interval, left- and right-hand responses were measured by asking subjects to perform a parity judgment task and give a response only to those numbers that belonged to the remembered sequence. The results clearly showed that the position of the number in the working memory sequence was strongly associated with space: numbers from the beginning of the sequence were responded to faster with the left hand than with the right hand and vice versa for numbers towards the end of the sequence. Number magnitude, on the other hand, was not systematically associated with left or right responses.

If position in working memory relates to response preference, it should be possible to establish this position-based effect with whatever kind of information maintained in working memory. Hence, in the next experiment van Dijck and Fias [39] used fruit and vegetable names and asked subjects to perform a fruit/vegetable classification task during the retention interval. Again, a position-based effect on response preference was observed. Interestingly, the SNARC effect was also measured in the same subjects, using a traditional parity judgment taks, i.e. without any working memory instructions. Crucially, the position-based effect with fruits and vegetables significantly correlated with the SNARC effect with numbers. This strongly suggests that the association between working memory position and space is what drives the SNARC effect, not the long-term numerical value.

These findings suggest that what happens is that in order to achieve efficient task performance participants store numbers in working memory as part of the task set and that, in doing so, they systematically order numbers as a function of their numerical magnitude, maybe as a helpful way of 
extending working memory capacity. From this explanation of the SNARC effect a number of phenomena can be readily explained. First, it enables to explain the fact that the SNARC effect is obtained when number magnitude is completely irrelevant for the task [30]. It is not the task that has to be performed that is crucial but if and how a number is systematically stored in working memory. Second, the range dependency of the SNARC effect $[30,5]$ is a consequence of the fact that only numbers that belong to the task set are stored. Third, the fact that the SNARC effect also occurs for non-numerical stimuli with an intrinsic ordinal structure [letters of the alphabeth, 40, musical tones, 41, 42, arbitrary stimui that have been learned to be ordered, 43, 44, 45] is not surprising, given that not numerical value but systematic assignment to a serial position in working memory is important. Fourth, mapping small numbers to the beginning and large numbers to the end of the memorized task set sequence is the default mapping. However, task context can change this default mapping. This is the case for instance when participants are asked to imagine numbers on a clock face [e.g. 31]. Similarly, the direction of the SNARC effect depends on reading habits (with a reversed SNARC effect in right-to-left reading cultures [with a reversed SNARC effect in right-to-left reading cultures, 46]. It has been shown that the direction of the SNARC effect can rapidly change within the same participants, depending on whether they read a text from left-toright or from right-to-left immediately before the measurement of the SNARC effect [35]. While this is hard to explain by a long-term oriented mental number line account, the positional WM account easily accounts for this flexibility, although at this point it is not clear whether reading direction determines how the numbers belonging to the task set are positionally coded in WM or, alternatively whether it is the position-space associations that are influenced by reading direction. 


\section{General discussion}

The present review of our recent work challenges the dominant view that the mental representation of numerical magnitude takes the form of a mental number line which is homeomorphic with the representation of physical lines and that there is a common spatial attention mechanism that operates both in physical space and in number space. We specifically considered the number interval bisection task in neglect and the SNARC effect, because these tasks have been considered prototypical exemplars of this homeomorphism.

Regarding the SNARC effect, our work generated results that are difficult to reconcile with the mental number line hypothesis. First, the spatial reponse code that is associated to numbers doesn't seem to be of a visuospatial nature but is rather situated at the level of conceptual categories. Second, serial position in working memory is the primary determinant of associations with response codes, not numerical magnitude. The fact that the same serial position effects also occur for non-numerical stimuli implies that the spatial associations that are observed in the SNARC effect are not specific to number but are a side effect of how serial position is coded in working memory and is related to spatial aspects of the response.

Also the single case study and the patient group study revealed evidence that is not in line with what is expected from the mental number line hypothesis. Both studies converge with previously made observations that number interval bisection and physical line bisection can be dissociated [e.g. 15, 16]. The single case study actually showed physical bisection biases to the left whereas the number interval bisection biased remained towards the larger number.

Moreover, these patient studies are also suggestive of working memory being a crucial determinant of the bisection bias. The single case study revealed that a position-specific deficit to initial items of the verbal working memory could explain the number interval bisection bias. The group study showed that the number interval bisection bias was not associated with line bisection, but with the SNARC effect. Because the SNARC effect is clearly determined by serial position in working memory and because the position-specific working memory impairment in the single case 
study concur, we hypothesize that serial position drives the correlation between number bisection bias and the SNARC effect, thereby strengthening our hypothesis (derived from a single case) that also in number bisection the crucial variable is serial position.

In sum, the empirical work reviewed above questions the two key assumptions of the mental number line hypothesis: the homeomorphism between number and physical space and the common mechanism of spatial attention operating in both number and physical space. Instead, we propose that it are spatial associations that are defined in conceptual terms that are associated to number and that it is not spatial attention operating on a mental number line, but a systematic coupling between number magnitude and ordinal position in working memory that constitutes the underlying mechanism. We realize that these are pointers towards an alternative framework but that we haven't reached the stage of a fully elaborated functional explanation yet.

Clearly, the SNARC effect and the number bisection bias are not the sole phenomena that are indicators of interactions between number and space that have been taken as support for the mental number line hypothesis. First, Fischer et al. [11] showed that numbers can act as an endogenous cue in the Posner cueing paradigm, suggesting that numbers can induce shifts of spatial attention. Second, using fMRI, Knops et al. [47] showed that the pattern of neural activity in regions that are involved in planning saccades could distinguish between subtraction and addition, with subtraction being associated with leftward eye-movements and addition with rightward eyemovements. Although the causal involvement needs to be demonstrated, it might suggest a close link between spatial attention and mental calculation. Third, Ishihara et al. [48] showed that number-induced manual pointing to various horizontal spatial positions was facilitated when the location corresponded to the position on the mental number line, suggesting a close connection between numbers and visuomotor processes. 
It might be that the mental number line is a valid hypothesis in these situations. At this point, however, we believe that any conclusions are premature in the absence of further testing. Given the fact that our work showed that the link between number and space is far more complex than we originally thought, a large body of work is needed to come to grips with this complexity.

In doing so, we might have to go beyond the boundaries that are typically drawn to demarcate research fields. For instance, how are spatial attention and working memory related? Hereby, recent work indicating an overlap in neural resources between attention and visuospatial working memory should be taken into account [e.g. 49]. A related question is whether spatial attention plays also a role in verbal working memory, as was recently suggested by Anderson et al. [50]. This in turn raises the old issue to what extent visual and verbal working memory are interrelated. Are they organized in a modality-specific way as in the model of Baddeley \& Hitch [51] or do they depend on modality-independent mechanisms [52, 53]. Of course, also insights on how serial order processing in working memory is accomplished may turn out to be extremely relevant [e.g. 52, 54, 55]. It might be particularly useful to connect to the models that are designed in the context of serial order processing in working memory [see 56 for a review]. Recently, Botvinick and Watanabe [57] explicitly made this connection by proposing a model on serial position in working memory that explicitly incorporated a mechanism that was based on number selective neural tuning [e.g. 58].

Similarly, insight can be gained from the recently growing research line that tries to understand how instructions are implemented and executed [e.g. 59, 60]. Because instructions are usually conveyed to the subject in verbal form, often referring to binary categorical labels as left or right (e.g. press left if even, press right if odd), one can ask the question whether this might be a causal factor for the dominance of the conceptual spatial representation in the SNARC effect. But, what would happen when instructions are not delivered in terms of linguistic spatial categories (press left or press right)? The study of Ishihara et al. [48] might be indicative in this respect. Relatedly, one should think about the role of the specific task to be performed. For instance, why is 
it that parity judgment is affected by verbal working memory and magnitude comparison by visuospatial working memory [38]?

Another important avenue for further research is to better understand the nature and organization of knowledge representations about abstract concepts like space and number. Clearly, this knowledge is initially constructed from sensorimotor experience (for instance number occupying space or order going in a specific direction). But gradually these experiences are summarized in abstract concepts (like small vs large; left vs right; or individual numbers) and linked to language. A crucial question in the current context is to what extent these abstract concepts keep the characteristics of the representations involved in sensorimotor experience and are thus embodied [e.g. 61] or, alternatively, become functionally separable from these concrete experiences [e.g. 62], possibly mediated by language [63]. One can then ask refined questions to what extent language-based conceptual knowledge versus knowledge more directly rooted in sensorimotor experience is invoked during number processing, probably depending on the nature of the task [e.g. 64 for a demonstration that exact addition relies on linguistic knowledge and approximate addition on visuospatial knowledge representations]

To conclude, having provided data that are incompatible with the mental number line hypothesis, our results may have raised more questions than that it has provided answers. It was a fruitful approach to push the mental number line metaphor to the limits of the idea that number shares a type of representation that is homomorphic to physical space with spatial attention operating upon it. Yet, now the limits of this hypothesis are becoming clear and alternative hypotheses including conceptual knowledge and serial order in working memory need to be considered to come to a complete understanding of the complexity of number-space interactions. Referring to the location of the 24th Attention and Performance meeting, one could use the French expression "Reculer pour mieux sauter" to indicate what is ahead of us now. 



\section{References}

1 Galton, F. (1880) Visualised numerals. Nature 21, 252-256

2 Seron, X., et al. (1992) Images of numbers, or when 98 is upper left and 6 sky blue. Cognition 44, 159-196

3 Kadosh, R.C. and Henik, A. (2007) Can synaesthesia research inform cognitive science? Trends in Cognitive Sciences 11, 177-184

4 Gevers, W., et al. (2010) Bidirectionality in Synesthesia Evidence From a Multiplication Verification Task. Experimental Psychology 57, 178-184

5 Dehaene, S., et al. (1993) The mental representation of parity and number magnitude. Journal of Experimental Psychology-General 122, 371-396

6 Fias, W. and Fischer, M.H. (2005) Spatial representation of number In Handbook of Mathematical Cognition (Campbell, J.I.D., ed), pp. 43-54, Psychology Press

7 Zorzi, M., et al. (2002) Brain damage - Neglect disrupts the mental number line. Nature 417, 138139

8 Halligan, P.W., et al. (2003) Spatial cognition: evidence from visual neglect. Trends in Cognitive Sciences 7, 125-133

9 Dehaene, S. (2001) Precis of the number sense. Mind Lang. 16, 16-36

10 Stoianov, I., et al. (2008) Visuospatial priming of the mental number line. Cognition 106, 770 779

11 Fischer, M.H., et al. (2003) Perceiving numbers causes spatial shifts of attention. Nature Neuroscience 6, 555-556

12 Umilta, C., et al. (2009) The spatial representation of numbers: evidence from neglect and pseudoneglect. Experimental Brain Research, 561-569

13 Hubbard, E.M., et al. (2005) Interactions between number and space in parietal cortex. Nature Reviews Neuroscience 6, 435-448

14 Vallar, G. and Girelli, L. (2009) Numerical representations: Abstract or supramodal? Some may be spatial. Behavioral and Brain Sciences 32, 354-+

15 Doricchi, F., et al. (2005) Dissociation between physical and mental number line bisection in right hemisphere brain damage. Nature Neuroscience 8, 1663-1665

16 Doricchi, F., et al. (2009) Spatial Orienting Biases in the Decimal Numeral System. Current Biology 19, 682-687

17 van Dijck, J.-P., et al. (under revision) Non-Spatial Neglect for the Mental Number Line.

18 Grossi, D., et al. (1989) On the different roles of the cerebral hemispheres in mental- imagery The oclock test in 2 clinical cases. Brain and Cognition 10, 18-27

19 Denis, M., et al. (2002) Visual perception and verbal descriptions as sources for generating mental representations: Evidence from representational neglect. Cognitive Neuropsychology 19, 97112

20 van Dijck, J.-P., et al. (2009) The internal structure of number space. In The cognitive mechanisms of spatial numerical associations (van Dijck, J.-P.), Unpublished doctoral disertation

21 Barsalou, L.W. (1999) Perceptual symbol systems. Behavioral and Brain Sciences 22, 577-+ 22 Paivio, A. (1986) Mental representations : a dual coding approach. Oxford university press

23 Kosslyn, S.M., et al. (1989) Evidence for 2 types of spatial relations - hemispheric specialization for categorical and coordinate relations. Journal of Experimental Psychology-Human Perception and Performance 15, 723-735

24 Kosslyn, S.M. (2006) You can play 20 questions with nature and win: Categorical versus coordinate spatial relations as a case study. Neuropsychologia 44, 1519-1523 
25 Priftis, K., et al. (2006) Explicit versus implicit processing of representational space in neglect: Dissociations in accessing the mental number line. Journal of Cognitive Neuroscience 18, 680-688

26 Proctor, R.W. and Cho, Y.S. (2006) Polarity correspondence: A general principle for performance of speeded binary classification tasks. Psychological Bulletin 132, 416-442

27 Gevers, W., et al. (2006) Numbers and space: A computational model of the SNARC effect. Journal of Experimental Psychology-Human Perception and Performance 32, 32-44

28 Santens, S. and Gevers, W. (2008) The SNARC effect does not imply a mental number line. Cognition 108, 263-270

29 Gevers, W., et al. (2010) Verbal-spatial and visuo-spatial coding of number-space interactions. Journal of Experimental Psychology-General 139, 180-190

30 Fias, W., et al. (1996) The Importance of Magnitude Information in Numerical Processing: Evidence from the SNARC Effect. Mathematical Cognition 2, 95-110

31 Bachtold, D., et al. (1998) Stimulus-response compatibility in representational space. Neuropsychologia 36, 731-735

32 Gevers, W., et al. (2006) Automatic response activation of implicit spatial information: Evidence from the SNARC effect. Acta Psychologica 122, 221-233

33 Notebaert, W., et al. (2006) Shared spatial representations for numbers and space: The reversal of the SNARC and the Simon effects. Journal of Experimental Psychology-Human Perception and Performance 32, 1197-1207

34 Marble, J.G. and Proctor, R.W. (2000) Mixing location-relevant and location-irrelevant choicereaction tasks: Influences of location mapping on the Simon effect. Journal of Experimental Psychology-Human Perception and Performance 26, 1515-1533

35 Shaki, S. and Fischer, M.H. (2008) Reading space into numbers - a cross-linguistic comparison of the SNARC effect. Cognition 108, 590-599

36 Shaki, S. and Gevers, W. (in press) Cultural characteristics dissociate magnitude and ordinal information processing. Journal of Cross-Cultural Psychology

37 Herrera, A., et al. (2008) The role of working memory in the association between number magnitude and space. Acta Psychologica 128, 225-237

38 van Dijck, J.-P., et al. (2009) Numbers Are Associated with Different Types of Spatial Information Depending on the Task. Cognition 113, 248-253

39 Van Dijck, J.-P. and Fias, W. (submitted) A working memory accout for spatial numerical associations.

40 Gevers, W., et al. (2003) The mental representation of ordinal sequences is spatially organized. Cognition 87, B87-B95

41 Lidji, P., et al. (2007) Spatial associations for musical stimuli: A piano in the head? Journal of Experimental Psychology-Human Perception and Performance 33, 1189-1207

42 Rusconi, E., et al. (2006) Spatial representation of pitch height: the SMARC effect. Cognition 99, 113-129

43 Previtali, P., et al. (2010) Placing order in space: the SNARC effect in serial learning. Experimental Brain Research 201, 599-605

44 Van Opstal, F., et al. (2009) The neural representation of extensively trained ordered sequences. Neuroimage 47, 367-375

45 Lourenco, S.F. (2010) Developmental Origins and the Extent of Generalization in the Representation of Magnitude. In 24th Attention and Performance meeting, July 6-10, Paris, France 46 Zebian, S. (2005) Linkages between number, concepts, spatial thinking, and directionality of writing: The SNARC effect and the reverse SNARC effect in English and Arabic monoliterates, biliterates, and illiterate Arabic speakers. Journal of Cognition and Culture 5, 165-190

47 Knops, A., et al. (2009) Recruitment of an Area Involved in Eye Movements During Mental Arithmetic. Science 324, 1583-1585

48 Ishihara, M., et al. (2006) Interaction between space and number representations during motor preparation in manual aiming. Neuropsychologia 44, 1009-1016 
49 Lepsien, J. and Nobre, A.C. (2007) Attentional modulation of object representations in working memory. Cerebral Cortex 17, 2072-2083

50 Anderson, E.J., et al. (2010) Overlapping functional anatomy for working memory and visual search. Experimental Brain Research 200, 91-107

51 Baddeley, A.D. and Hitch, G. (1974) Working memory. In The psychology of learning and motivation (Bower, G.H., ed), pp. 47- 90, CA: Academic Press

52 Majerus, S., et al. (2010) The commonality of neural networks for verbal and visual short-term memory. Journal of Cognitive Neuroscience 22, 2570-2593

53 Nystrom, L.E., et al. (2000) Working memory for letters, shapes, and locations: fMRI evidence against stimulus-based regional organization in human prefrontal cortex. Neuroimage 11, 424-446

54 Majerus, S., et al. (2006) The left intraparietal sulcus and verbal short-term memory: Focus of attention or serial order? Neuroimage 32, 880-891

55 Marshuetz, C., et al. (2000) Order information in working memory: fMRI evidence for parietal and prefrontal mechanisms. Journal of Cognitive Neuroscience 12, 130-144

56 Marshuetz, C., et al. (2006) Working memory for order and the parietal cortex: An event-related functional magnetic resonance imaging study. Neuroscience 139, 311-316

57 Botvinick, M. and Watanabe, T. (2007) From numerosity to ordinal rank: a gain-field model of serial order representation in cortical working memory. Journal of Neuroscience 27, 8636-8642

58 Nieder, A., et al. (2002) Representation of the Quantity of Visual Items in the Primate Prefrontal Cortex. Science 297, 1708 - 1711

59 Brass, M., et al. (2009) Neural Correlates of Overcoming Interference from Instructed and Implemented Stimulus-Response Associations. Journal of Neuroscience 29, 1766-1772

60 Waszak, F., et al. (2008) Cross-talk of instructed and applied arbitrary visuomotor mappings. Acta Psychologica 127, 30-35

61 Lakoff, G. and Johnson, M. (1999) Philosophy in the Flesh: The embodied mind and its challenge to Western thought. Basic Books

62 Boroditsky, L. and Ramscar, M. (2002) The roles of body and mind in abstract thought. Psychological Science 13, 185-189

63 Boroditsky, L. (2003) Linguistic Relativity. In Encyclopedia of Cogntive science (Nadel, L., ed), pp. 917- 921, MacMillan Press

64 Dehaene, S., et al. (1999) Sources of mathematical thinking: Behavioral and brain-imaging evidence. Science 284, 970-974

65 Srinivasan, M. and Carey, S. (2010) The long and the short of it: On the nature and origin of functional overlap between representations of space and time. Cognition 116, 217-241

66 Chen, Q. and Verguts, T. (2010) Beyond the mental number line: A neural network model of number-space interactions. Cognitive Psychology 60, 218-240

67 Hedden, T., et al. (2008) Cultural influences on neural substrates of attentional control. Psychological Science 19, 12-17

68 Noël, M.-P. (2010) Spatial numerical associations in preschoolers. In 24th Attention and Performance meeting, July 6-10, Paris, France

69 Casasanto, D. and Boroditsky, L. (2008) Time in the mind: Using space to think about time. Cognition 106, 579-593

70 Imbo, I., et al. (submitted) The development of the SNARC-effect: Evidence for early verbalspatial coding.

71 Dehaene, S. and Cohen, L. (2007) Cultural recycling of cortical maps. Neuron 56, 384-398

72 Dehaene, S., et al. (2008) Log or linear? Distinct intuitions of the number scale in western and amazonian indigene cultures. Science 320, 1217-1220 
73 Núñez, R. (in press) No Innate Number Line in the Human Brain. . Journal of Cross-Cultural Psychology

74 de Hevia, M.D. and Spelke, E.S. (2010) Number-Space Mapping in Human Infants. Psychological Science 21, 653-660 


\section{Box 1. The origin of number space interactions}

Despite the compelling demonstrations of an intimate relation between the representations of space and numbers, two questions are still outstanding. Both pertain to the origin of the number space interactions. The first question is a more general one: Why is it that both domains are associated at a functional and neural level? The second is more specific and asks why there is a systematic directional preference in this association (why is a small number associated with left and a large number with right).

At least three possible explanations have emerged in the literature [e.g. 65]. A first view holds that the functional overlap between numbers and space derives from experienced correlations between numbers and space [e.g. 5, 66]. In our daily life, children often see numbers in typical and culturally consistent spatial configurations (e.g. on blackboards, in schoolbooks, on computer keyboards) and it is a well established observation that such environmental and cultural factors are picked up and can have an influence on both behavioral and brain organization [e.g. 67]. Since these lay-outs of number are systematically organized in space (e.g. from left-to-right in Western cultures), this view naturally addresses both questions. Indeed, it has been shown that the direction of functional overlap between numbers and space critically depends on the direction of the reading system (which also determines the orientation in which number sequences are most common spatially depicted) of the tested subjects, whereby left-to-right readers associate small numbers with left and large numbers with right, and right- to left readers in the opposite direction $[46,35]$. A recent study showed culturally dependent directions of number space associations in preschoolers [68], suggesting that it is not reading direction per se but the culturally consistent organization of spatial lay-outs of ordinal information that are important.

A second view is that both domains are related to one another because of the shared vocabulary to talk about spatial and numerical entities (e.g. five is bigger than four, six is smaller than nine). It is 
assumed that during development of their language skills children pick up this equivalence, thereby determining and constraining the conceptual representation of both numbers and space [e.g. 69]. This view provides an answer to the first question but, without further assumptions, is largely silent regarding the nature of the specific directionality of the number space association. In line with this view, it has been recently demonstrated that the SNARC-effect is the expression of a congruency between verbal concepts rather than between numbers and a location in physical space [29, 38]. A recent developmental study showed that such a conceptual relationship is already the dominant factor driving the SNARC-effect in third grade children, the youngest group tested [70].

A third and final view holds that the link between numbers and space is innate in the sense that it is the result of a process of recycling evolutionary old general-purpose mechanisms (like spatial processing) for more recent cognitive skills like number processing [e.g. 71]. For instance, Dehaene and colleagues [72] investigated the mental representation of numbers in an Amazonian tribe. Notwithstanding the fact that the members of this tribe lack a fully developed lexicon for numbers and formal (math) education, they spontaneously put numbers on a horizontal line when asked to map numbers to space [but see 73]. Other support for the native origin has been provided by de Hevia and Spelke [74] who demonstrated number-space interactions in nine month old infants, ruling out the idea that language acquisition and extensive experienced correlations between numbers and space are crucial for the emergence of a relation between both domains. So far, this account did not provide much evidence for the directional specificity issue.

In sum, it is clear that the origin of the relationship between numbers and space is multifaceted and cannot be reduced to one single mechanism. To the contrary, it seems that the functional overlap between both domains illustrates how environmental, cultural and linguistic experience can shape an innate biological tendency to link different domains of cognition. Our recent experiments (see main text) suggest that serial working memory position could be a crucial intermediate variable that determines how environment, culture and language effectuate the number space relationship. It is not unlikely that language and cultural experience structure the spatial organization of serial 
position in working memory and provide the systematicity in which ordinal information is mapped to positions in working memory. It is also reasonable to assume that number processing recruits and recycles the general-purpose resources provided by working memory because of its ordinal organization which it shares with the number system. 


\section{Figure Captions}

\section{Figure 1: SNARC-effect}

When asked to categorize numbers as a function of parity status or numerical magnitude, humans who read from left to right, have the tendency to respond faster to small numbers with a left response, and faster to large numbers with a right response. The effect is called the Spatial Numerical Associations of Response codes (SNARC) effect. Here we depict the SNARC effect of both the parity judgment and magnitude comparison task, by presenting the observed data and the regression lines, representing RT differences between right and left-hand responses in function of the numerical magnitude. Positive values reflect faster left-hand than right- hand responses. The presented data reflects the effects of the baseline conditions described in van Dijck et al. [38].

\section{Figure 2: Physical line and number interval bisection}

To test the hypothesis that the mental representation of numbers takes the form of a left to right oriented line, the relation between numbers and space has been investigated in right brain damaged neglect patients suffering from an attentional deficit for the left side of space. It was reasoned that when numbers were actually represented on such a representational format, their attentional deficit should give rise to a similar rightward bias when bisecting the mental number line as when indicating the midpoint of actual physical lines [7]. Here we present the results of a physical line and a number interval bisection task administered to neglect patients and two control groups [20], showing that neglect patients indeed shift the subjective midpoint of a numerical interval to the right (i.e. a positive deviation on the figure). This close resemblance in the performance of both tasks, led to the conclusion that the mental number line is more than a metaphor, and that its representational format is functionally isomorphic to that of physical lines [e.g. 7, 13]. 
Figure 1

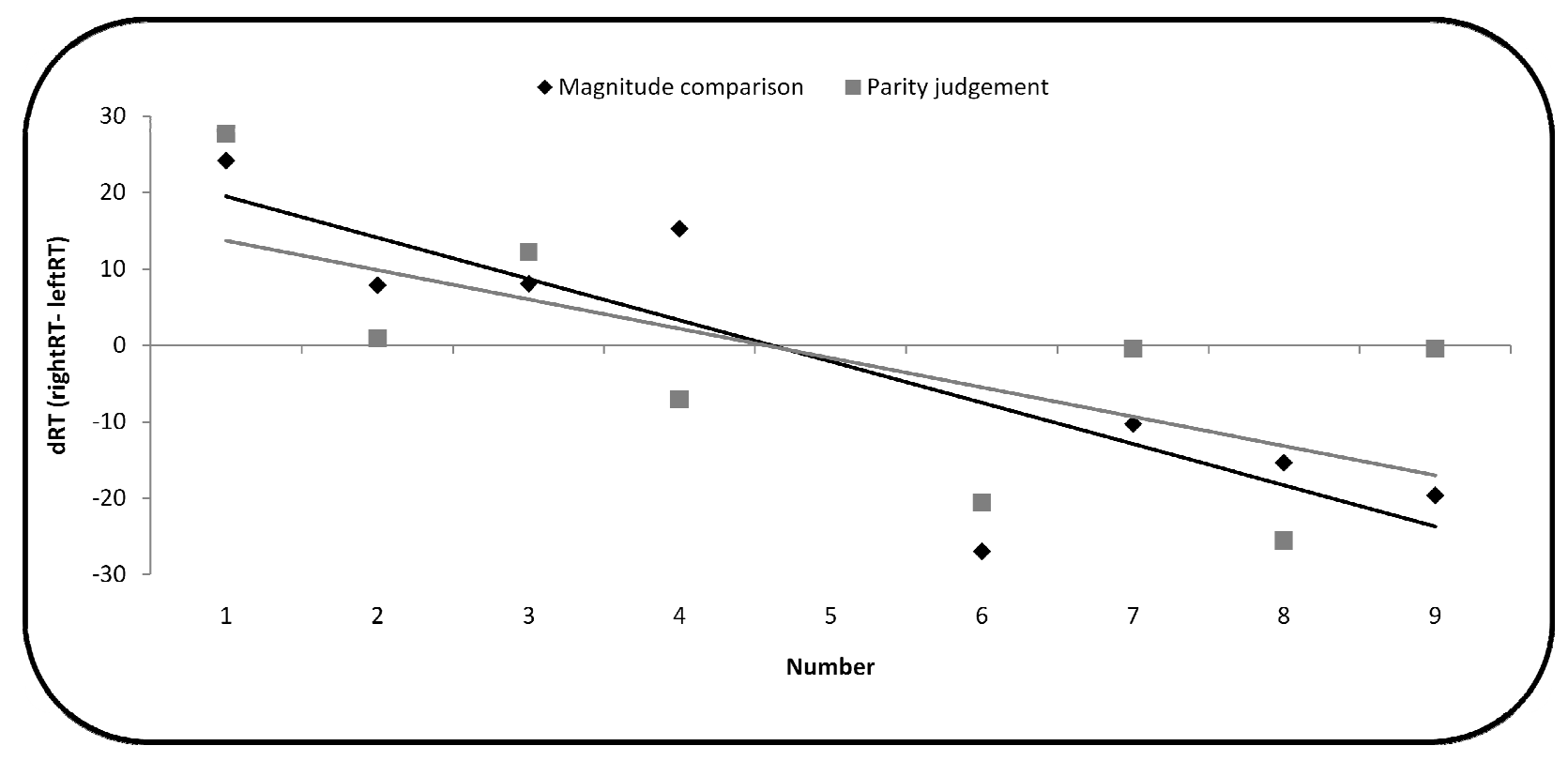


Figure 2

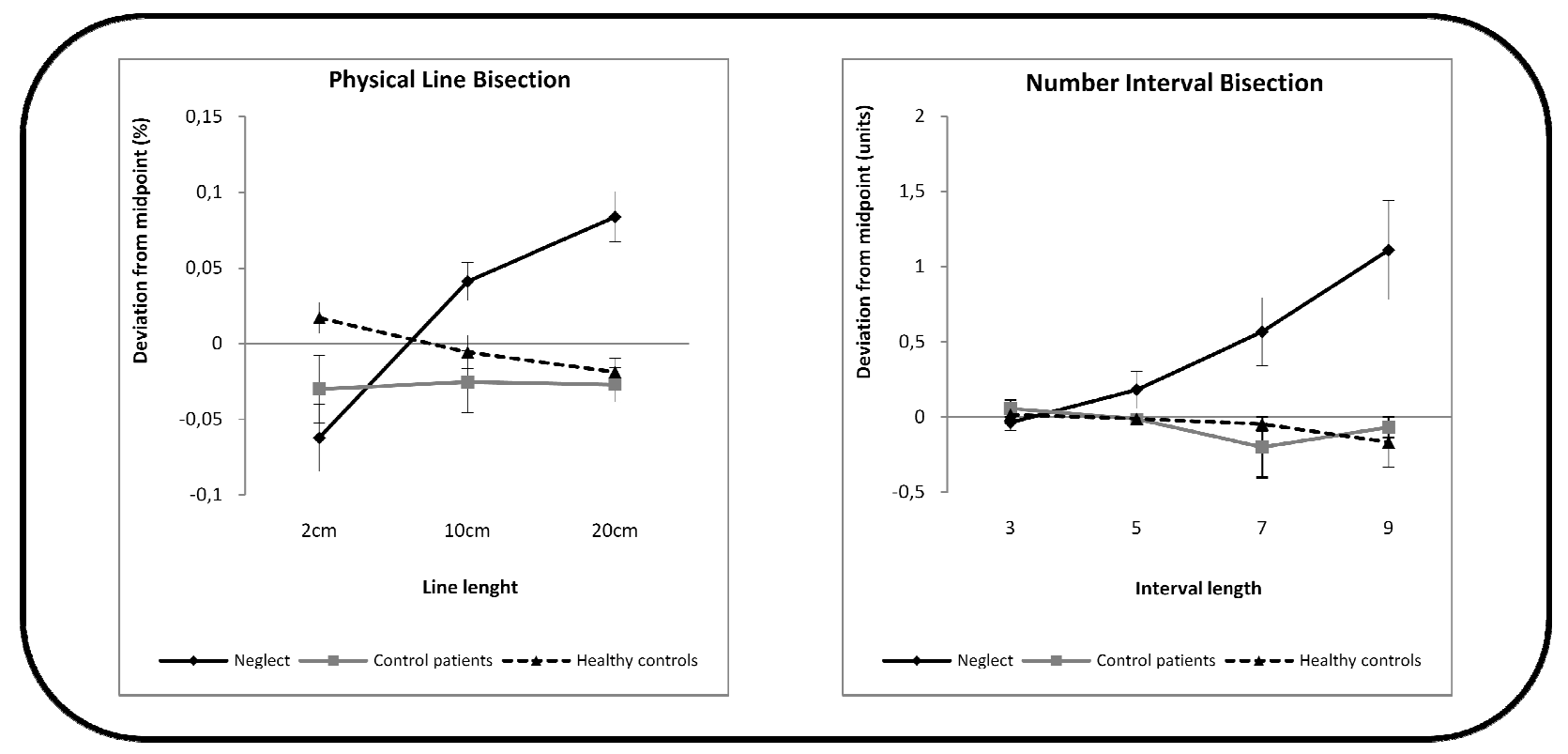

